\title{
Observer based dynamic control model for bilaterally controlled MU-LapaRobot: surgical tool force limiting
}

\author{
Branesh M. Pillai ${ }^{1}$, Chumpon Wilasrusmee ${ }^{2}$, Jackrit Suthakorn ${ }^{3}$ \\ ${ }^{1,3}$ Department of Biomedical Engineering, Faculty of Engineering, Mahidol University, Thailand \\ ${ }^{2}$ Department of Surgery, Faculty of Medicine Ramathibodi Hospital, Mahidol University, Thailand
}

\begin{tabular}{|c|c|}
\hline Article Info & ABSTRACT \\
\hline Article history: & During laparoscopic surgeries, primary surgical tool insertion is \\
\hline Received Apr 22, 2019 & the demanding and strenuous task. As the surgeon is unaware of the type of \\
\hline Revised Sep 20, 2019 & ease the procedure, the movement of the surgical tool needs to be controlled. \\
\hline Accepted Sep 27, 2019 & $\begin{array}{l}\text { It's the operational capabilities that are to be manipulated to perform } \\
\text { a smooth surgery even from a distant location. In this study, a robot system is }\end{array}$ \\
\hline Keywords: & $\begin{array}{l}\text { being introduced for laparoscopic primary surgical tool insertion. It will } \\
\text { incorporate a novel observer based dynamic control along with robot assisted }\end{array}$ \\
\hline Bilateral control & bilateral control. Moreover, a virtual spring damper force lock system is \\
\hline Force control & $\begin{array}{l}\text { introduced through which the slave system will notify the master regarding } \\
\text { the target achieved and excessive force. The validation of the proposed }\end{array}$ \\
\hline Haptics & control system is experimented with bilaterally controlled MU-LapaRobot. \\
\hline Medical robotics & The experiment is comprising 3 cases of bilateral control criteria which are \\
\hline Motion control & non-contact motion, contact motion, and limit force locking. The results \\
\hline Mu-Laparobot & defined the same value for contact and non-contact motion by $0.3 \mathrm{~N}$. \\
\hline Robotics surgery & $\begin{array}{l}\text { The results depicted a force error of } 3.6 \% \text { and a position error of } 5.8 \% \text { which } \\
\text { validated the proposed algorithm. }\end{array}$ \\
\hline
\end{tabular}

Copyright @ 2020 Institute of Advanced Engineering and Science. All rights reserved.

Corresponding Author:

Jackrit Suthakorn,

Center for Biomedical and Robotics Technology (BART LAB),

Department of Biomedical Engineering, Faculty of Engineering, Mahidol University,

999, Phuttamonthon Sai 4, Salaya, Nakorn Pathom, 73170, Thailand.

Email: jackrit.sut@mahidol.ac.th

\section{INTRODUCTION}

Minimally Invasive Surgery (MIS) was initially conducted in 1980's and was the first laparoscopic cholecystectomy [1-3]. Since then many modifications have been proposed to technically enhance the skill of surgeons [4-6]. MIS has its set of limitations that relate to the technical and mechanical nature of the equipment, loss of haptic feedback (force and tactile), etc. [7]. At present the instruments have restricted degrees of motion; most of them have 4 degrees of motion, whereas the human wrist and hand have 7 degrees of motion. In addition to this, surgeons experience lack of direct palpation, poor visibility due to no direct viewing of organs, poor hand-eye coordination, meager information about the depth, no proper force feedback from touching the organs and motion constraints through small incision [8-12]. Furthermore, trembling of hands while conducting MIS is also a matter of immense concern in terms of tissue injury. These problems motivated a number of research groups from engineering field to develop the system that facilitates the surgeons with these problems.

During Laparoscopic surgeries, primary surgical tool insertion provides an access to entry portal. It is a crucial step and requires precision and accuracy. Primary surgical tool associated insertions might cause injuries to gastrointestinal walls or to the blood vessels [13-15]. The trembling of hands or the clumsiness at the part of the surgeon leads to such injuries. Thus, to conduct primary surgical tool insertion a staff member with hands on experience is a must, subject to availability [16]. Therefore, 
the rationale for developing surgical robots is to overcome the limitations of current laparoscopic technologies. Robots have high accuracy and precision and can work with stability without continuous tremor effect. It is controlled remotely and provides minimally invasive access [17-20]. It works as a navigational aid and is unaffected by radiation. The experiments conducted by researchers at John Hopkins University, established that tremor is maximum when the experiment is carried by hand whereas it is controlled and minimized with the application of robots [8].

Robotic assistance is a feasible and a compact way for overcoming the gaps regarding human functionality. Such drawbacks and gaps are observed in fine, or dexterous manipulation tasks. Fine manipulation tasks involve high precision micrometer-level positioning accuracy. Natural factors such as physiology tremor that occurs in all normal, active muscle and drift are some of the factors in establishing these limitations [20]. Tremor further increases with stress, anxiety and physical exertion. Robotic systems are inherently unaffected by these factors. Keeping in view the shortcomings of the available robot systems, in the current study, robot based primary surgical tool insertion system is introduced by incorporating a novel observer based dynamic control along with robot assisted bilateral controlled laparoscopic surgery. The problem with a bilaterally controlled robotic system along with a redundant task is that it operates by using both systems simultaneously and therefore, often results into uncontrolled internal configuration of the system $[21,22]$. Thus, when dealing with such systems, it is much feasible to use dynamics. As they are dynamically uncoupled, both the parameters i.e. task and posture can be controlled independently. This method will enhance the operational capabilities of robotic system. Bilateral Control Systems works in accordance with action and reaction principle as shown in Figure 1.

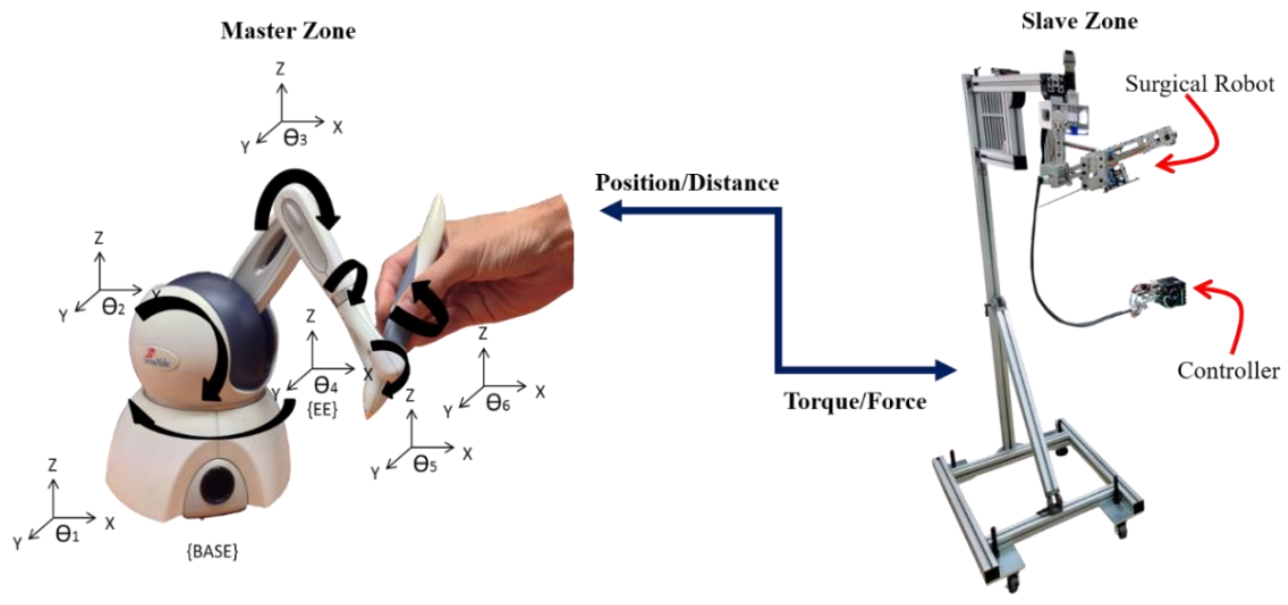

Figure 1. Bilaterally controlled robotic system

The operator operates via master device onto the environment through slave device [23-26]. Operator delegates command to the slave device via master device. Master device not only acquires motion feedback but also the amount of force to be exerted on the environment. So, the force sensor when added to the slave device will transmit reaction forces. The force sensors have limited bandwidth [27]. Moreover, the disturbance observer (DOB) is incorporated as a torque sensor and is effective against disturbance compensation. Furthermore, it accounts for reaction torque estimation [28-30]. Therefore, the force controller will determine the reaction torque while sensing the internal disturbance of the system by increasing the cutoff frequency.

Furthermore, a virtual spring-damper force lock system is also introduced [31]. With the aid of this system the slave side will provide feedback to the master side to avoid excessive force exertion. This feedback is sent via flexible elastic feel observed at master's side. Virtual spring controller is inserted at master side and force lock is affianced at slave's side. Moreover, with addition of virtual damper control system the absence of position of the actual force that is applied from master manipulator is equalized and is compensated [32]. In this study we are focusing on improving the Operational capability of the Bilaterally Controlled MU-LapaRobot primary surgical tool insertion. In the said case the motion of the system is segregated into constrained direction and unconstrained direction. This is achieved via controlling the speed of surgical tool. The position, orientation and the region of the tool tips relative to each other vary the speed and internal organs in patient's abdomen based on the robot's workspace. Surgeon needs to focus on the target (operating point), environment (sensitive tissues in the operating regions) and about the collision of 
tools while conducting surgical procedure [33]. In this study the core concept is to control the movement of surgical robot during laparoscopic primary surgical tool insertion, so as to avoid extra burden on the surgeon to think if the robotic arm and tool will go beyond the operating region, or the tools will go below the operating parts or is there any collision between the tools and gastrointestinal organs. The work flow of the perspective procedures is depicted in Figure 2.

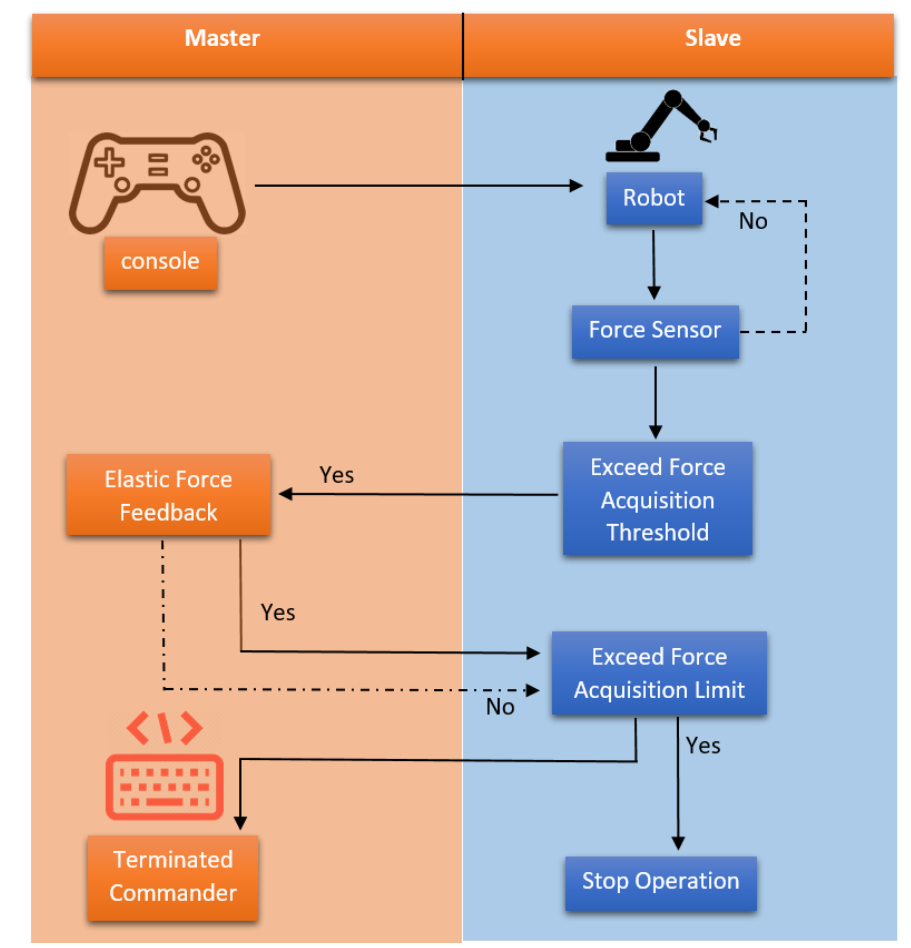

Figure 2. Experimental work flow of the perspective procedure

\section{MATERIALS AND METHODS}

\subsection{Developed surgical robotic system}

MU-LapaRobot is a surgical robotic system that assists in minimally invasive surgery [34]. This robot can generate 4 degree-of freedoms (DOFs) via remote fixed point called remote center of motion $(\mathrm{RCM})$ as shown in Figure 3. End effector side of the robot is installed with force sensor and surgical tool is manipulated by wire-driven transmission from driving controller. Wire-driven transmission is helpful for designing the following parameters: size, weight and load for actuators. In driving unit actuators and electromagnetic clutches transmit torque from actuators to robot. In collaborative operation, robot is able to perform as a surgical tool holder via manual adjustments and as a control in bilateral operation.

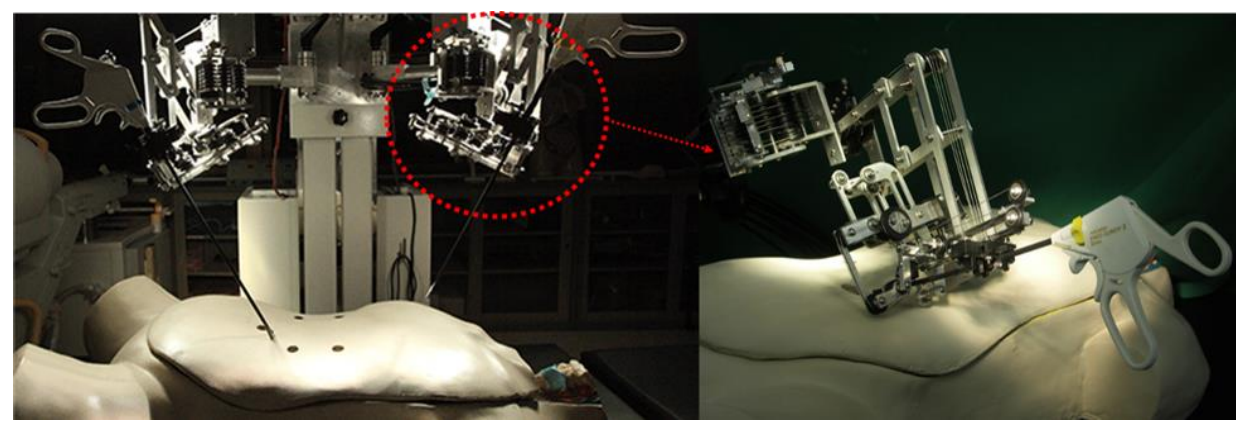

Figure 3. Bilaterally controlled MU-LapaRobot 
The bilaterally controlled MU-LapaRobot is simple in design and can be used easily by any surgeon without spending much time on training. MU-LapaRobot require less area and can save enough space in the operating room. This robot uses parallelogram mechanism and haptic omni device used at operator side. It takes a little volume and is lightweight due to its design. MU-LapaRobot will be helpful in terms of assistance during operation.

\subsection{Dynamics of sensorless based observatory task control}

The control system concerning redundant task experiences complexity primarily caused by the lack of control of posture. Adhering to the fact, fundamental dynamics (Newton's Second Law) is relatively more suitable, enabling the independent control of task and posture [22]. Here, the task and posture are taken to be controlled independently, where task and posture tracking errors respectively are defined as

$$
\begin{array}{ll}
E_{\theta}\left(\theta, \theta^{r e f}\right)=\theta(q)-\theta^{r e f}, & E_{\theta}\left(\theta, \theta^{r e f}\right) \in R^{P X 1} \\
E_{P}\left(\theta_{P}, \theta_{P}^{r e f}\right)=\theta_{P}-\theta_{P}^{r e f}, & E_{P}\left(\theta_{P}, \theta_{P}^{r e f}\right) \in R^{(n-P) X 1}
\end{array}
$$

In the above equations $\theta^{r e f}$ and $\theta_{P}{ }^{r e f}$ refers to the task and posture references. Consequently, the dynamics of the system can be written in following forms

$$
\begin{aligned}
& \ddot{E}_{\theta}=\ddot{\theta}-\theta^{r e f}=\delta_{\theta}^{-1}\left\{\tau_{\theta}-\left\{\delta_{\theta} J A^{-1}(D+G)-\delta_{\theta} \dot{J} \dot{\theta}\right\}\right\}-\ddot{\theta}^{r e f} \\
& \ddot{E}_{P}=\ddot{\theta}_{P}-\ddot{\theta}_{P}^{r e f}=\delta_{P}^{-1}\left\{\tau_{P}-\left\{\delta_{P} \Gamma_{P} A^{-1}(D+G)-\delta_{P} \dot{\Gamma}_{P} \dot{\theta}\right\}\right\}-\ddot{\theta}_{P}^{r e f} \dot{\theta}^{r e f}
\end{aligned}
$$

In closed loop, the task and posture control error dynamics can be expressed in following forms

$$
\begin{aligned}
& \ddot{E}_{\theta}+K_{d \theta} \dot{E}_{\theta}+K_{P \theta} E_{\theta}=0^{P X P} \\
& \ddot{E}_{P}+K_{d P} \dot{E}_{P}+K_{P P} E_{P}=0^{(n-P) X(n-P)}
\end{aligned}
$$

The closed loop task error dynamics in the above equations are defined by the design parameters $K_{d \theta}, K_{d P} \in R^{P X P}$, whereas, $K_{d P}, K_{P P} \in R^{(n-P) X(n-P)}$ represents the design parameters in the posture control loop. After these parameters are chosen independently, the calculation of desired acceleration can be performed as shown in equations below:

$$
\begin{aligned}
& \ddot{E}^{d e s}=\ddot{E}^{r e f}-\left(K_{d \theta} \dot{E}_{\theta}+K_{P \theta} E_{\theta}\right) \\
& \ddot{\theta}^{d e s}=\ddot{\theta}^{r e f}-\left(K_{d P} \dot{E}_{P}+K_{P P} E_{P}\right)
\end{aligned}
$$

The desired acceleration for task and posture control has the same structure. Since the desired accelerations are obtained, the task force can be written as

$$
\begin{aligned}
& \tau_{\theta}=\left[\delta_{\theta} J A^{-1}(D+G)-\delta_{\theta} \dot{J} \dot{\theta}\right]+\delta_{\theta} \ddot{\theta}^{d e s}=\tau_{\theta d e s} \delta_{\theta} \ddot{\theta}^{d e s} \\
& \tau_{P}=\left[\delta_{P} \Gamma_{P} A^{-1}(D+G)-\delta_{P} \dot{\Gamma}_{P} \dot{\theta}\right]+\delta_{P} \ddot{\theta}^{d e s}=\tau_{P d e s} \delta_{P} \ddot{\theta}_{P}^{\text {des }}
\end{aligned}
$$

Analogous to the desired acceleration, control forces as well demonstrate a similarity in structure for both the task control and the posture control. Here, matrices $\delta_{\theta}$ and $\delta_{P}$ represents disturbances in task and position controls respectively. In configuration space, when disturbances are estimated, consequently the term (D+G) in both (7) and (8) becomes zero. Hence, the task control torque could be rewritten as (9)

$$
\tau_{\theta}=\delta_{x} \dot{J} \dot{\theta}+\delta_{\theta} \ddot{\theta}^{d e s}
$$


Similarly, the posture control torque could be rewritten as

$$
\tau_{\theta}=\delta_{x} \dot{\Gamma}_{P} \dot{\theta}+\delta_{P} \ddot{\theta}_{P}^{\text {des }}
$$

From (9) and (10) the configuration space torque can then be expressed as

$$
T=J^{\tau} \tau_{\theta}+\Gamma_{P}^{\tau} \tau_{P}
$$

Considering the compensated configuration space disturbances $J^{-1} \theta \ddot{\equiv} T$ and substituting (9), (10) and (11) into (6), the desired acceleration of operation space becomes (12).

$$
\ddot{\theta}^{d e s}=J^{\#}\left(\ddot{\theta}^{d e s}-\dot{J} \dot{\theta}\right)+\Gamma_{P}^{\#}\left(\ddot{\theta}_{P}^{\text {des }}-\dot{\Gamma}_{P} \dot{\theta}\right)
$$

where $J^{\#}=A^{-1} J^{T} \delta_{\theta}$ represents the task Jacobian pseudoinverse and $\Gamma^{\#}=A^{-1} \Gamma_{P}{ }^{T} \delta_{P}$ represents the posture Jacobian pseudoinverse. The result complies with the constrained system control, and in place of posture controlling another task, it offers an approach of combining task and posture control.

Through the implementation of disturbance observers, the components of the control forces can be determined. Apparently, the structural blocks are similar to that of constrained system control, which results in query regarding task-constraint relationship in multibody system. Besides, the structure plays a vital role when designing the control for multibody system which interacts with environment or performs cooperative tasks. As shown in Figure 4, the control in operational space and enforcement of constraints forms a similar structure. This demonstrates a degree of similarity in multibody systems regarding the soft constraints and the redundant task control. On that basis, a system which may be constrained but requires the realization of a certain task performs the motion control. Hence, the equations representing the dynamics of constrained system and tasks have consistency regarding velocities and forces. This allows the combination of constraints and tasks in wider scope and they can be treated based on same framework.

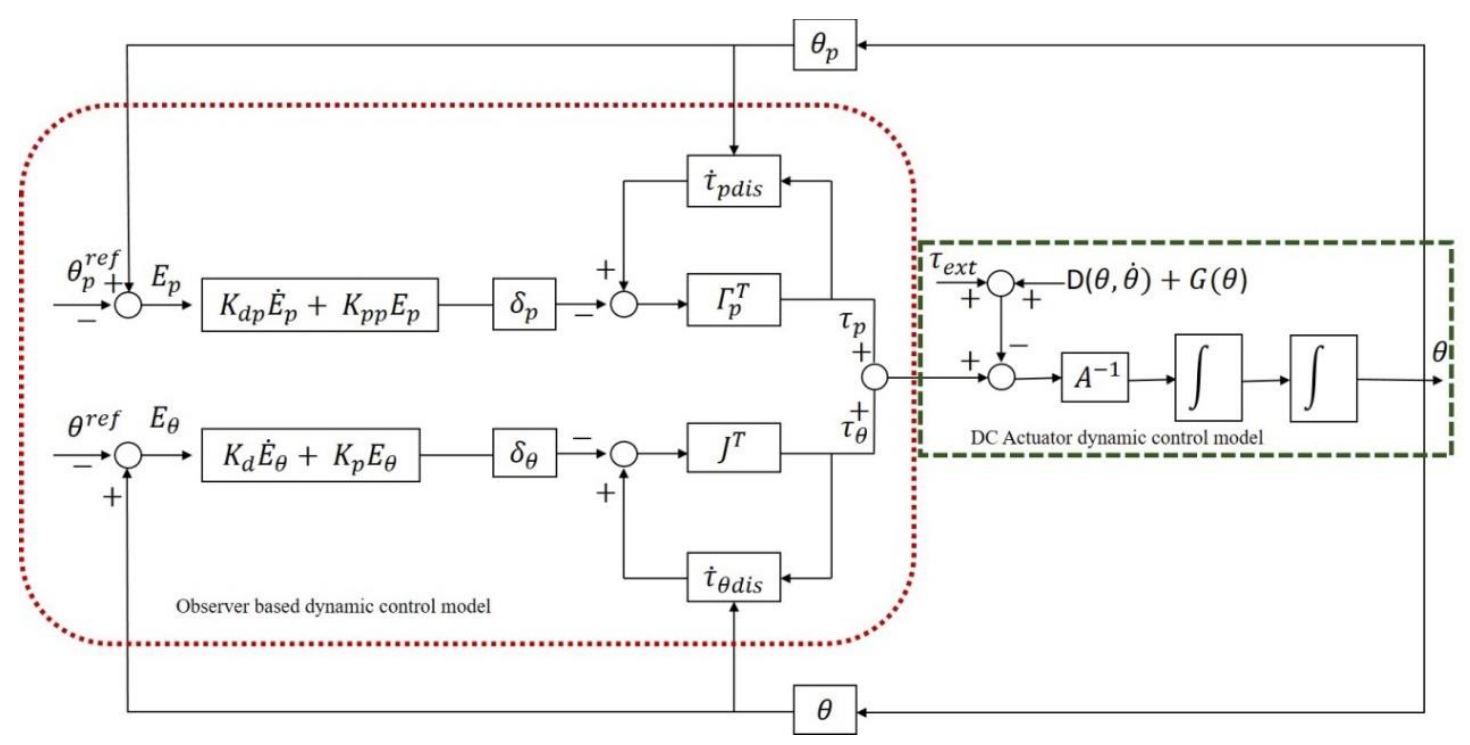

Figure 4. Observer based dynamic control model

\subsection{Virtual spring damper controller}

A conventional bilateral control system is signified by the integration of a virtual spring system in order to reduce the vibration problem. As the slave manipulator approaches a firm surface, master manipulator experiences resistance which impedes the motion. When extra force is applied, slave manipulator starts to tremble, disturbing the overall operation of the system. The spring controller incorporates a simple torsional spring, damper and two masses which are synchronized as shown in Figure 5. 


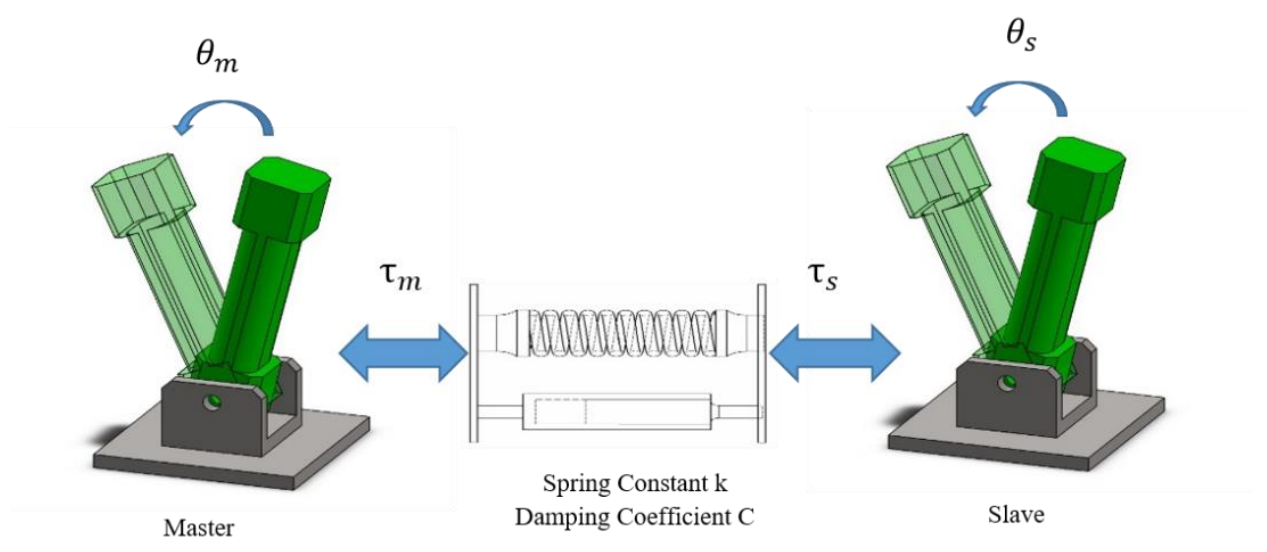

Figure 5. Virtual spring damper controller

$k$ : Spring constant of the virtual spring; $c$ : Damping coefficient of the virtual spring; $\theta_{m}$ : Angular displacement of master manipulator; $\theta_{s}$ : Angular displacement of slave manipulator; $\tau_{s}$ : External torque acting on the slave manipulator; $\tau_{m}$ : External torque acting on the master manipulator. $\tau_{m}$ and $\tau_{s}$ are provided by "master" and "slave" actuated torques through the tele-operated manipulators, providing equal and opposite torques through the human operator and environment. In order to implement in the control block diagram, the (13) and (14) were adopted from Harsha et al. [35]. For master,

$$
\left(J_{m} s^{2}+c s+k\right) \theta_{m}=T_{m}+(k+c s) \theta_{s}
$$

For slave,

$$
\left(J_{m} s^{2}+c s+k\right) \theta_{s}=(k+c s) \theta_{s}-T_{s}
$$

Furthermore, when an operator performs primary surgical tool insertion, the system significantly reduces the elastic effect. The use of spring effect smoothens the operator's experience and augments the efficiency by significantly reducing any chances of operator feeling nervous due to the system. In addition, as the slave operator approaches any object, an input of small deflection is produced through the change in spring constant corresponding to the nature of object. This will further enhance the comfort of the operator associated with the device. Moreover, surgeon can preset the surgical tool force limit before the beginning of bilateral control operation. The modeling of the reaction force pertaining to the feedback of the surgical tool can be constructed as

$$
\tau=J \ddot{\theta}+B \dot{\theta}+k \theta
$$

The Figure 6 outlines the overall complete functional force controller block diagram. The reference force is considered to be the operator preset force whereas the force sensor feedback is taken as the reaction force. As force error is calculated, it is fed back to the system in form of current through the PD controller. In Figure $6 \tau_{m}{ }^{e s t}, \tau_{s}{ }^{e s t}$ are the estimated disturbance torque by DOB and summed with commanded torque to attain the systems actual torque. 


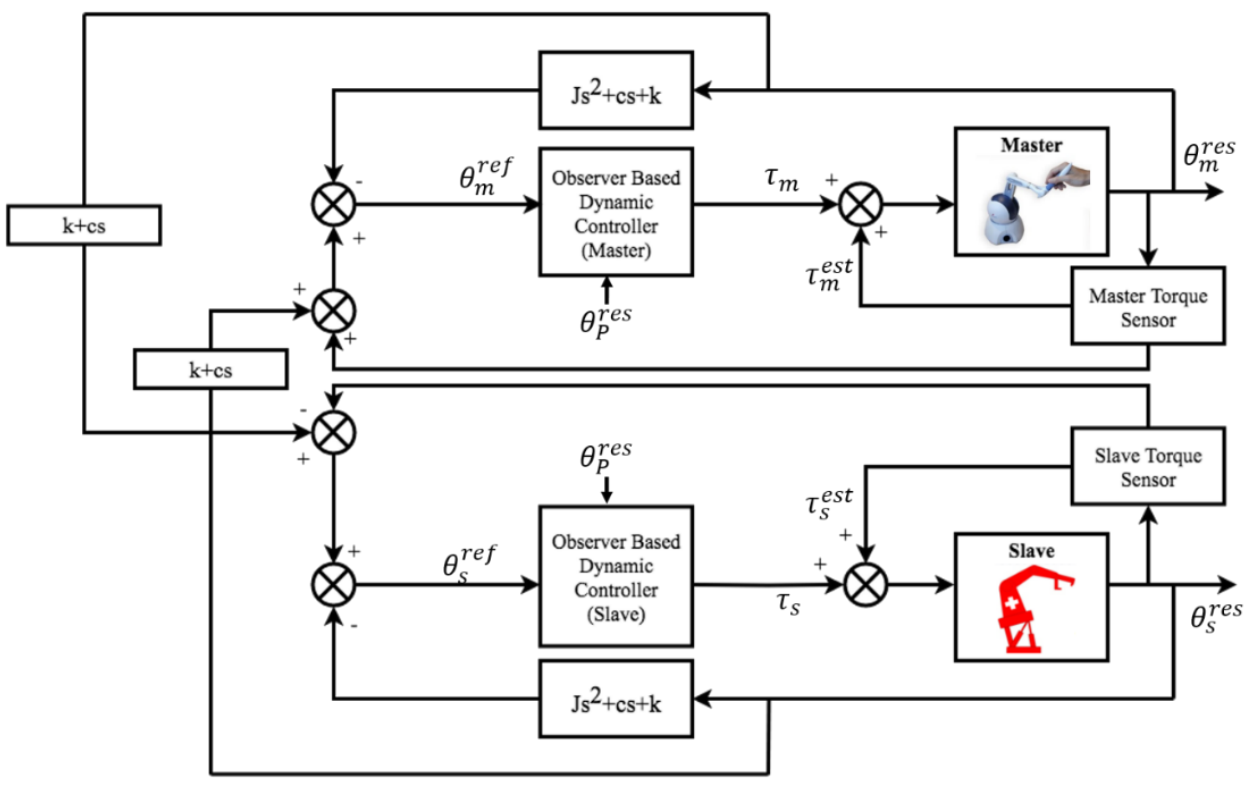

Figure 6. Overall complete functional force controller block diagram

\section{EXPERIMENTS AND RESULTS}

The MU-LapaRobot is a surgical system and is setup as a bilateral control as shown in Figure 7. The slave side of the MU-LapaRobot comes into contact with the working environment i.e. patient; the surgeon on the master side increases the working torque. When the slave tool is locked, bilateral controller starts to unfasten and notifies an elastic force for a predefined period. This is received at the surgeon's side in the form of elastic feel indicating that reaction force limit has been achieved. If the operator further exerts excessive force on the master surgical tool, a virtual spring damper controller is activated. At this point, resting indicator is copied from the MU-LapaRobot position. In the said scenario, force locking on the slave side will be activated. Due to fix force the gripped tissue deforms or gets damaged.

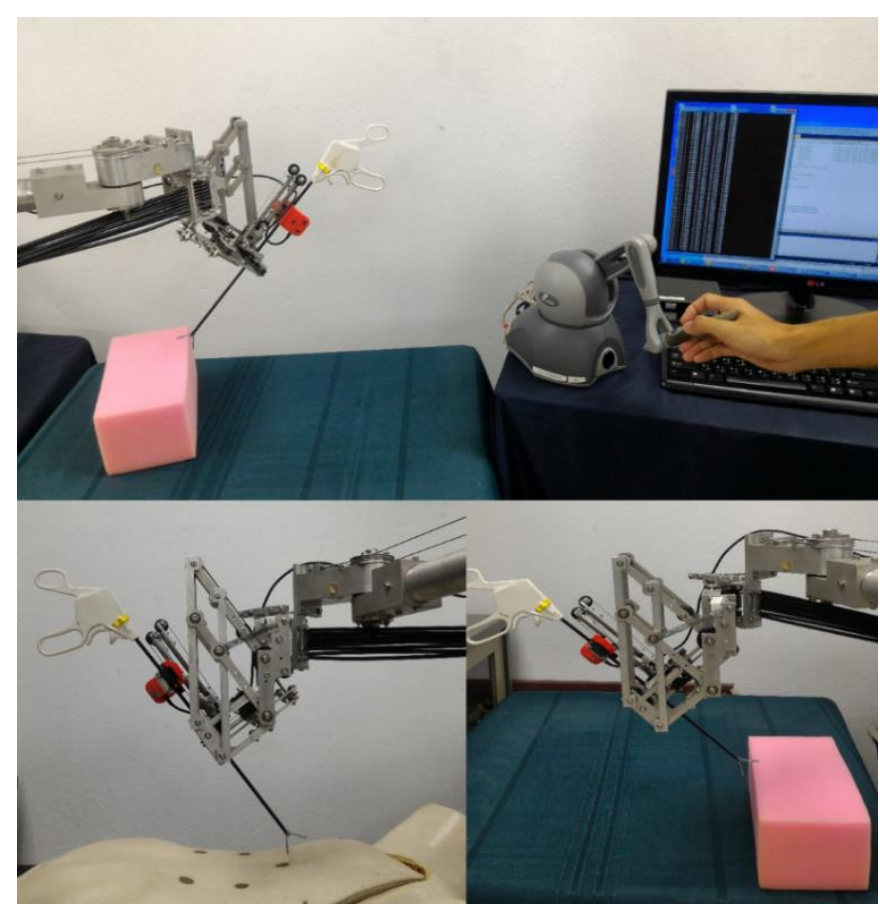

Figure 7. Bilaterally controlled MU-LapaRobot as an experimental setup 
On slave side, the MU-LapaRobot is composed of four Maxon motors integrated with $512 \mathrm{ppr}$ encoder resolutions. Three actuators are used for three rotational joint movements and one translational joint movement. The actuators are driven and controlled using Maxon EPOS2 controller which provides PID control in each motor. On the master side, the haptic device (Phantom Omni) is used to manipulate the end effector. The haptic device consists of six degree of freedoms (DOFs) and provides 3 dimensional force feedback to the stylus. Therefore, the system is implemented using 4 joints of haptic device (master side) to control the joint movement of MU-LapaRobot's slave side.

The experiment considers the bilateral control of surgical tool insertion which provides force feedback to master side. The robot's slave side is controlled remotely by haptic device which will generate the force in specific workspace. The contact force from the surgical tool insertion into the tissue and position of surgical tool at the slave side is measured along the contact motion. The experiment is comprising 3 cases of bilateral control criteria which are non-contact motion, contact motion, and limit force locking. In noncontact motion- the master side controls the slave side. In addition to this, there is a predefined force threshold for force acquisition of slave side. In this scenario, surgeon cannot feel the force feedback from haptic device. Next scenario is a contact motion in which the force on slave side is increased over the initial force threshold and master side can sense an elastic force feedback as reaction force. In this stage, the elastic force is depending on the displacement of surgical tool insertion that equals to master operation. In last case, the force limit is the maximum force threshold and is defined to avoid the damage to contact area. When the acquired force from robot's slave side is higher than the value of force limit; the robot will not continue operation and then stop. Similarly, master side will not feel the elastic force back anymore. As a result, for safety the master side on haptic device will change from generating force made to free moving.

Each experiment case has three different maximum force thresholds: $1 \mathrm{~N}, 1.5 \mathrm{~N}$, and $2 \mathrm{~N}$ subsequently. All the experiments define the same value between non-contact and contact motion by $0.3 \mathrm{~N}$ as an initial force threshold. In practical environment the tensile value of the human tissue varies from patient to patient, and thus the experiments are conducted with 2 types of materials sponge and rubber (with an unknown tensile strength value $15 \times 7 \mathrm{~cm}$ ) this experiment was performed under the supervision of a Surgeon from Ramathibodi Hospital, Mahidol University as shown in Figure 7. In order to check the stability of the proposed system, the materials with unknown tensile strength were utilized and this system can be applied universally to robot assisted laparoscopic surgeries.

The experiment is conducted by using a couple of rotary motors of the equal specification along with a one-DOF motion control test. Some of the relevant parameters of the experiment are tabulated in Table 1. Here, it is assumed that the spring constant of the object relies upon the pressing of the object and it represents the angle. The relation of the applied force from the master and the response from the slave along with the region of operation are demonstrated in Figure 8 (b), (d), (f) and Figure 9 (b), (d), (f). As shown, during the initial 5 seconds, force is applied by the master in order to overcome the system friction. While the slave is moved $3 \mathrm{~cm}$, it is in contact with the object. Eventually, the master increases the force reaching the maximum level. The preset value of force limit of the object can be assumed as $1 \mathrm{~N}, 1.5 \mathrm{~N}$, and 2 N. In regards to the slave force response, Figure 8 (b), (d), (f) and Figure 9 (b), (d), (f) shows that slave force reached the preset threshold value in different time interval. At that moment, despite the master changing the force profile without reversing the force, the slave force remains at predefined set value due to the activation of the force controller.

Table 1. Experimental parameters

\begin{tabular}{lccl}
\hline Parameters & Symbol & Value & Units \\
\hline Nominal Inertia & $J_{n}$ & 13.5 & $\mathrm{gcm}^{2}$ \\
Torque coefficient & $K_{t}$ & 0.256 & $\mathrm{Nm} / \mathrm{A}$ \\
Spring constant & $K_{x}$ & 0.5 & -- \\
Proportional constant & $K_{p}$ & 0.01 & -- \\
$\begin{array}{l}\text { Derivative constant } \\
\begin{array}{l}\text { Cut-off frequency of low pass } \\
\text { filter used in DOB as reaction } \\
\text { force sensor }\end{array}\end{array}$ & $K_{d}$ & 0.006 & -- \\
\hline
\end{tabular}


The object is released and bilateral control is retained when the master reverses the force also the master position response becomes less than or equal to $99 \%$ of the slave position response in non-contact motion. The master position response and the slave position response are demonstrated in Figure 8 (a), (c), (e) and Figure 9 (a), (c), (e) respectively. In contact motion mode, the system is accelerating while there is contact between slave and the object. The object is compressed by the slave up to its force limit due to the force applied on the master. At the force limit, the activated force controller maintains the slave position taking into account the object property it remains unchanged unless the master applies the releasing force. The position of the master during the force locked period on the slave is controlled by a spring controller which is appended onto the master. Apart from the force lock mode, master and slave operate under bilateral control. As the object is released, master and slave retain the bilateral control, system relying upon the applied force on the master.

In experiment, the results of master-slave control are shown separately in terms of position and force applied. The slave position after seizing of the contact motion observes a little change in reverse direction. The duration of the contact motion is in depend on the amount of force. The speed of master manipulation is corresponding with the time to obtain a force value. The force at the side of contact motion is increasing gradually after it is stopped at the defined maximum forces in both materials. The results defined the same value for contact and non-contact motion by $0.3 \mathrm{~N}$. The results as shown in Figure 10 depicted a force error of $3.6 \%$ and a position error of $5.8 \%$ estimated using both the standard linear regression method and the method of evaluation [36] which validated the proposed algorithm.
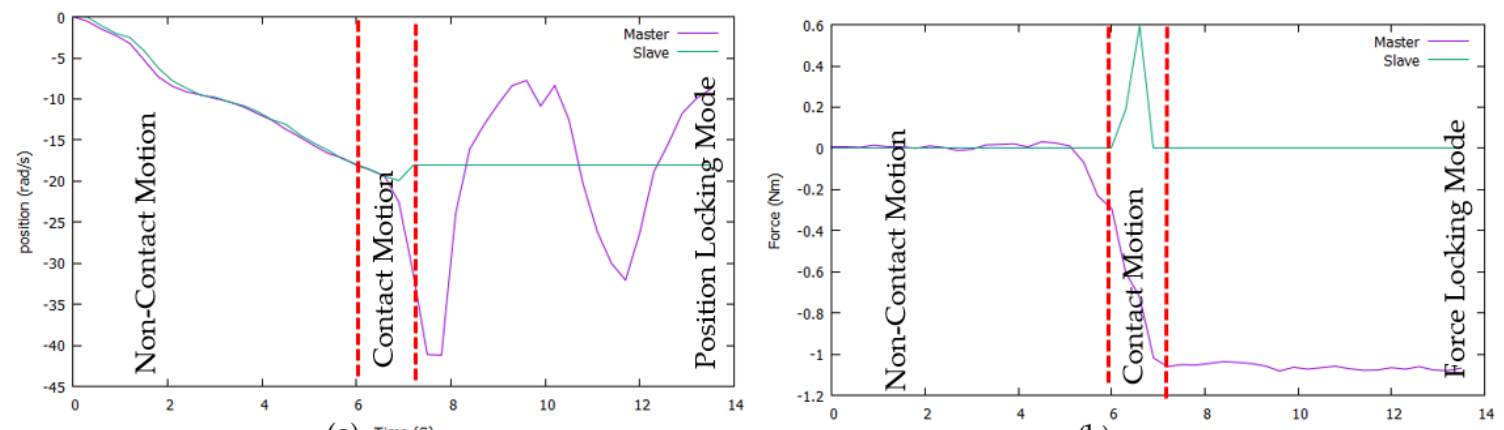

(a) Time (S)

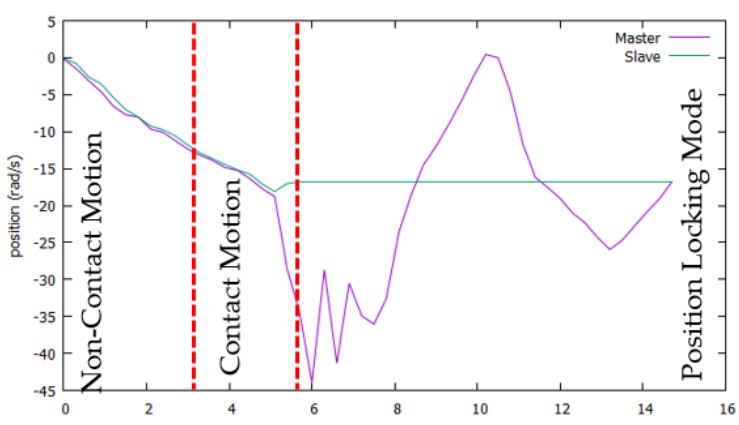

(c) Time (s)

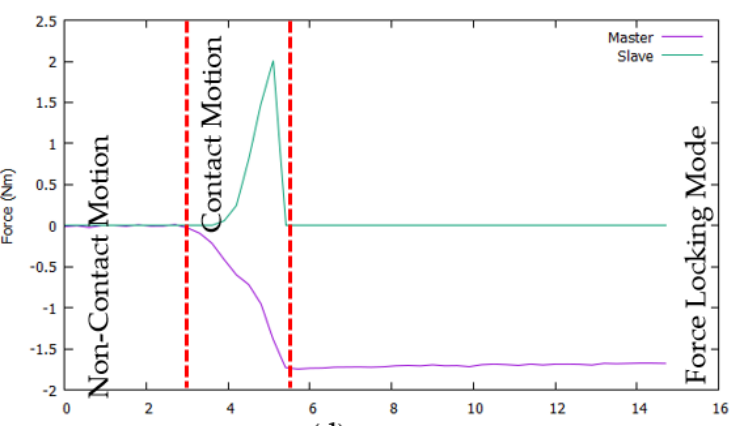

(d) Time (s)

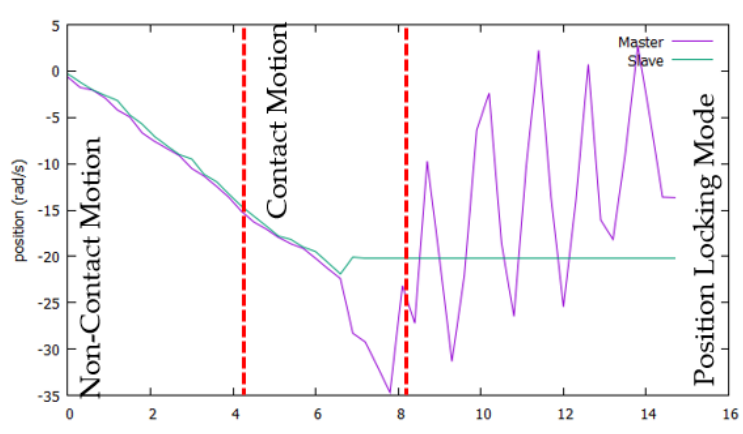

(e) Time (s)

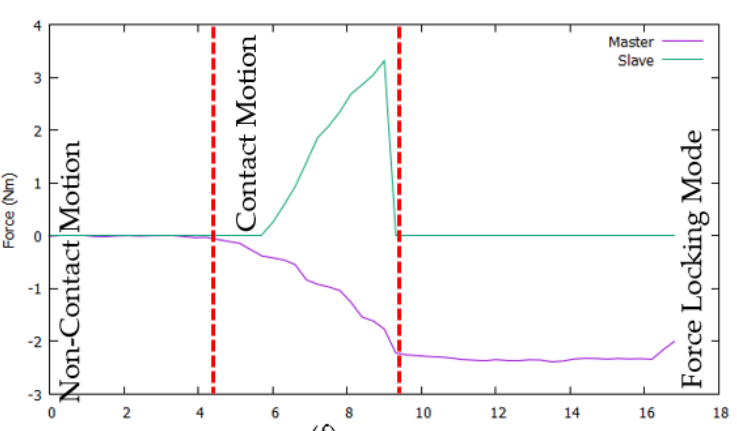

(f) Time (s)

Figure 8. Experiments and responses on rubber material (a) $1 \mathrm{~N}$ position (b) $1 \mathrm{~N}$ force (c) $1.5 \mathrm{~N}$ position (d) $1.5 \mathrm{~N}$ force (e) $2 \mathrm{~N}$ position (f) $2 \mathrm{~N}$ force 

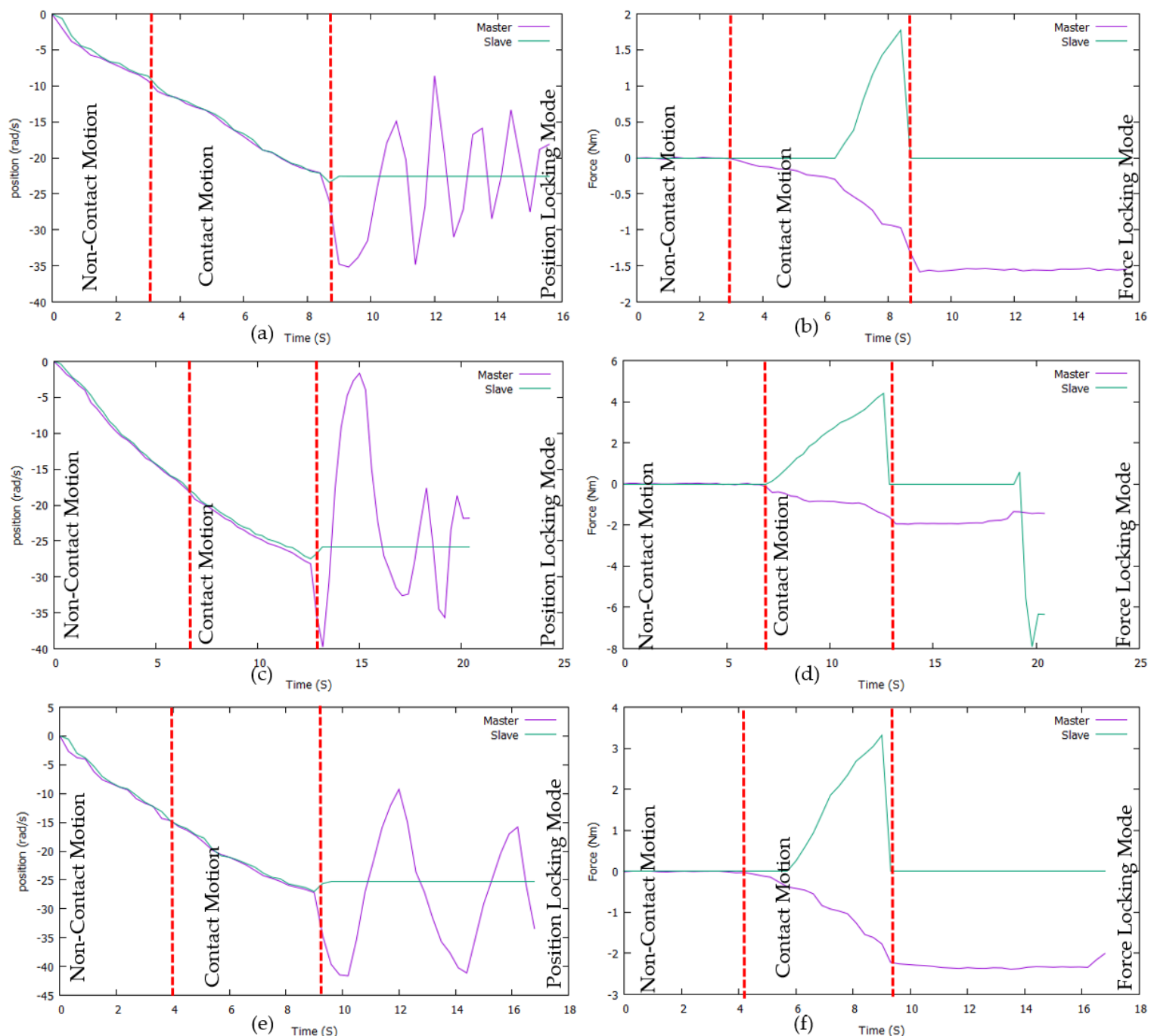

Figure 9. Experiments and responses on sponge material (a) $1 \mathrm{~N}$ position (b) $1 \mathrm{~N}$ force (c) $1.5 \mathrm{~N}$ position (d) $1.5 \mathrm{~N}$ force (e) $2 \mathrm{~N}$ position (f) $2 \mathrm{~N}$ force
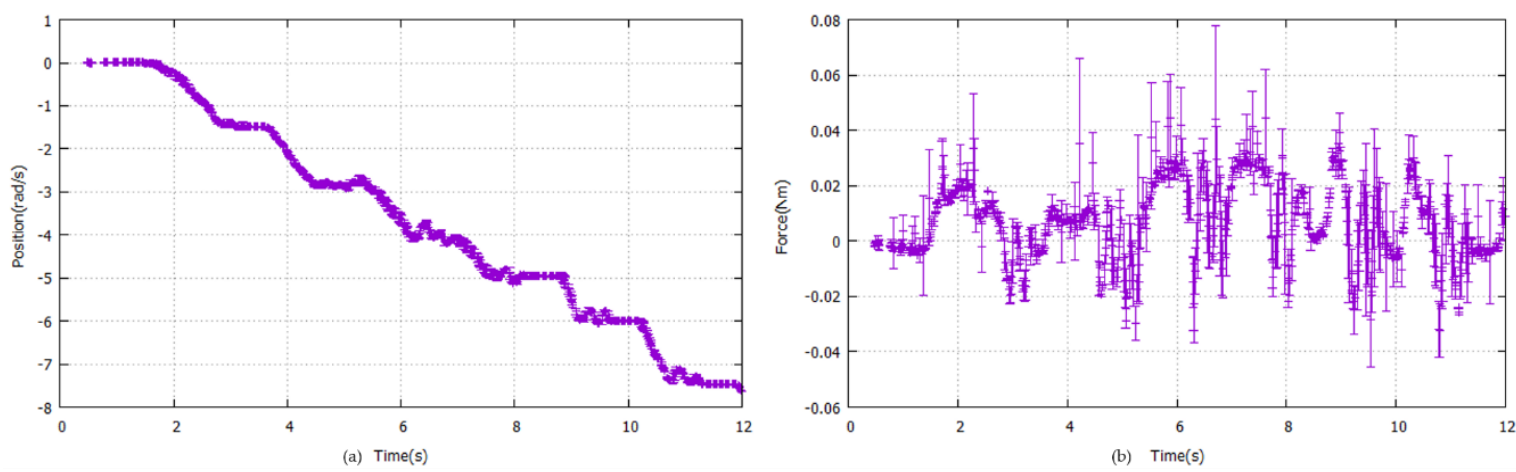

Figure 10. Error response analysis (a) position (b) force

\section{CONCLUSION}

In this paper, force limiting mechanism was incorporated with the bilateral control system. The proposed control system was applied for a single DOF translational motion at the slave side (surgical tool side). In course of safety the force limiting control system protected tissues from damage or unwanted 
insertion by the surgical tool. The achievement of the preset force was notified to surgeon at master side through spring back force. The master operator could sense the reaction force in addition to the spring effect. The position of bilateral control used with MU-LapaRobot is not stopped at the same time. This is due to the time delay of data communication which generated an extra force on slave side. Error compensation and estimation are needed to be implemented in future [37, 38]. In the force preset, parameters can be changed in a way that to compensate tissue stiffness variations. As a result, the force limit of the tissue is preset by the surgeon based on the experience and the material properties. Furthermore, force lock protects the tissue at the slave side and the threshold force limit is felt by the surgeon in the form of elasticity. The operator sets the force limit for the tissue based on his experience and properties of the tissue. Virtual spring controller system aligns the master-slave positions in the locked mode. Moreover, in this study the virtual model improves stability of the system. As explained in results, the responses recorded for master and slave operations are slightly varying but overall are significant for real time applications. The surgeon at the master side could feel a strong spring effect as reaction force via slave side. The range of control model parameters set in the wat that was not distorted tactile sensation and was able to conduct virtual model actions.

\section{ACKNOWLEDGEMENTS}

This research is financially support by National Research University Funds through Mahidol University and Government Research Budget through Mahidol University (Grant No. 111-2558). Another fund resource by the Computer-Integrated Intelligent Medical System Project under the Thailand National Research University Grant through Mahidol University, Thailand. Moreover, Sakol Nakdhamabhorn, Chawaphol Direkwatana, Syed Saqib Hussain Shah and BART LAB Researchers for their kind support.

\section{REFERENCES}

[1] R. Taylor, et al., "A Steady-Hand Robotic System for Microsurgical Augmentation,” The International Journal of Robotics Research, vol. 18, pp. 1201-1210, 1999.

[2] A Polychronidis, et al., "Twenty years of laparoscopic cholecystectomy: Philippe Mouret- March17, 1987," Journal of the Society of Laparoendscopic Surgeons, vol. 12, pp. 109-111, 2008.

[3] W. Rogers, et al., "Identifying surgical innovation: a qualitative study of surgeons' views," Ann Surg., vol. 259, pp. 273-278, 2014.

[4] W. J. Lee, et al., "Recent advances in laparoscopic surgery,” Asian J Endosc Surg., vol. 6, pp. 1-8, 2013.

[5] K. Hiramatsu, et al., "Can laparoscopic appendectomy be safely performed by surgical residents without prior experience of open appendectomy?" Asian Journal of Surgery, vol. 41, pp. 270-273, 2018.

[6] S. Y. Kim, et al., "Learning curve for a laparoscopic appendectomy by a surgical trainee," J Korean Soc Coloproctol, vol. 26, pp. 324-328, 2010.

[7] B. Yi, et al., "The first clinical use of domestically produced Chinese minimally invasive surgical robot system "Micro Hand S," Surg Endosc., vol. 30, pp. 2649-2655, 2016.

[8] R. H. Taylor, et al., "Medical Robotics and Computer-Integrated Surgery," in Siciliano B. and Khatib O. (eds), Springer Handbook of Robotics, Springer Handbooks, Springer, Cham, 2016.

[9] S. Hoteya, et al., "Feasibility and safety of laparoscopic and endoscopic cooperative surgery for gastric submucosal tumors, including esophagogastric junction tumors," Dig. Endosc, vol. 26, pp. 538-544, 2014.

[10] N. M. Galvao, et al., "Single port laparoscopic access surgery," Tech Gastrointest Endosc, vol. 11, pp. 84-93, 2009.

[11] H. G. Stassen, et al., Engineering for Patient Safety: Issues in Minimally Invasive Procedures, CRC Press, pp. 2-18, 2004.

[12] E. M. McDougall, et al., "Comparison of three dimensional and two-dimensional laparoscopic video systems," J Endourol, vol. 10, pp. 371-374, 1996.

[13] D. B. O'Connor and D. C. Winter, "The role of laparoscopy in the management of acute small-bowel obstruction: A review of over 2,000 cases," Surg Endosc, vol. 26, pp. 12-17, 2012.

[14] D. L. Warner and K. C. Sasse, "Technical Details of Laparoscopic Sleeve Gastrectomy Leading to Lowered Leak Rate: Discussion of 1070 Consecutive Cases,” Minimally Invasive Surgery, vol. 2017, 2017.

[15] J. Nagata, et al., "A novel transperitoneal abdominal wall nerve block for postoperative pain in laparoscopic colorectal surgery," Asian Journal of Surgery, vol. 41, pp. 417-421, 2018.

[16] N. Enayati, et al., "Haptics in robot-assistedsurgery: Challenges and benefits," IEEE Rev Biomed Eng, vol. 9, pp. 49-65, 2016.

[17] L. C. George, et al., "Residency Training in Robotic General Surgery: A Survey of Program Directors," Minimally Invasive Surgery, vol. 2018, 2018.

[18] M. Kroh, et al., "First human surgery with a novel single-port robotic system: cholecystectomy using the da Vinci Single-Site platform," Surg Endosc, vol. 25, pp. 566-73, 2011.

[19] M. Tonutti, et al., "The role of technology in minimally invasive surgery: state of the art, recent developments and future directions," Postgraduate Medical Journal, vol. 93, pp. 159-167, 2017.

[20] A. M. H. S. Abeykoon and K. Ohnishi, "Virtual tool for bilaterally controlled forceps robot-for minimally invasive surgery," The International Journal of Medical Robotics and Computer Assisted Surgery, vol. 3, pp. 271-280, 2007. 
[21] H. J. Marcus, et al., "Endoscopic and keyhole endoscope-assisted neurosurgical approaches: a qualitative survey on technical challenges and technological solutions," Br J Neurosurg., vol. 28, pp. 1-5, 2014.

[22] A. Sabanovic and K. Ohnishi, Motion control systems, IEEE press John Willey and sons (Asia) pte Ltd, First Edition, 2011.

[23] A. M. Okamura, "Methods for haptic feedback in tele operated robot assisted surgery," Industrial Robot, vol. 31, pp. 499-508, 2004.

[24] K. Ohnishi, et al., "Motion Control for Real-World Haptics," IEEE Trans. Ind. Electron. Magazine, vol. 4, pp. 16-19, 2010.

[25] A. M. H. S. Abeykoon and M. B. Pillai, "RTOS based embedded controller implementation of a bilateral control system," Journal of the National Science Foundation of Sri Lanka, vol. 42, pp. 217-228, 2014.

[26] S. A. Ali, et al., "The Effect of Parameters Variation on Bilateral Controller," International Journal of Power Electronics and Drive System (IJPEDS), vol. 9(2), pp. 648-659, 2018.

[27] S. Katsura, et al., "Analysis and Experimental Validation of Force Bandwidth for Force Control," IEEE Trans. Ind. Electron., vol. 53, pp. 922-928, 2006.

[28] B. M. Pillai and J. Suthakorn, "Motion control applications: observer based DC motor parameters estimation for novices," International Journal of Power Electronics and Drive System (IJPEDS), vol. 10(1), pp. 195-201, 2019.

[29] E. Saito and S. Katsura, "A filter design method in disturbance observer for improvement of robustness against disturbance in time delay system," IEEE Industrial Electronics (ISIE), pp. 1650-1655, 2012.

[30] M. A. M. Obaid, et al., "Robust Backstepping Tracking Control of Mobile Robot Based on Nonlinear Disturbance Observer," International Journal of Electrical and Computer Engineering (IJECE), vol. 6(2), pp. 901-908, 2016.

[31] R. M. M. Ruwanthika and A. M. H. S. Abeykoon, "Simulation of Enhanced Force Limiting Gripper for Bilateral Teleoperation," Proc. IEEE ICIAfS., pp. 1-6, 2014.

[32] A. M. H. S. Abeykoon and K. Ohnishi, "Improvement of Tactile Sensation of a Bilateral Forceps Robot by a Switching Virtual Model," Advanced Robotics, vol. 22, pp. 789-806, 2008.

[33] J. Yadav and J. Suthakorn, "Euclidean Distance and Workspace Region Based Control Algorithm for Collision Avoidance in a Laparoscopic Surgical Robot: MU-LapaRobot," Proc. IEEE ROBIO, pp. 2056-2061, 2012.

[34] C. Direkwatana and J. Suthakorn, "Development of Wire-Driven Laparoscopic Surgical Robotic System, MULapaRobot," Proc. IEEE ROBIO, pp. 485-490, 2011.

[35] M. K. Madawala, et al., "Virtual Torsional Spring Based Bilateral Control System for Soft Manipulation," Proc. IEEE ICCPCT, pp. 337-343, 2013.

[36] M. Kušnerová, et al., "A Proposal for Simplifying the Method of Evaluation of Uncertainties in Measurement Results," Measurement Science Review, vol. 13, pp. 1-6, 2013.

[37] N. S. Hasan, et al., "Harmonic Suppression of Shunt Hybrid Filter using LQR-PSO based," International Journal of Electrical and Computer Engineering (IJECE), vol. 7(2), pp. 869-876, 2017.

[38] Y. Li, "Stabilization of Teleoperation Systems with Communication Delays: An IMC Approach," Journal of Robotics, vol. 2018, 2018. 